\title{
Experimental study on cyclic tensile behaviour of air-entrained concrete after frost damage
}

\author{
JIAYI YUAN ${ }^{1},{\mathrm{XUDONG} \mathrm{CHEN}^{1, *^{(0)}} \text { and SHENGSHAN GUO }}^{2}$ \\ ${ }^{1}$ The College of Civil and Transportation Engineering at Hohai University, Nanjing, China \\ ${ }^{2}$ China Institute of Water Resources and Hydropower Research, Beijing 10048, China \\ e-mail: jy_yuan028@126.com; cxdong1985@hotmail.com
}

MS received 3 September 2018; revised 21 May 2019; accepted 7 July 2019

\begin{abstract}
To investigate fatigue tensile behaviour of air-entrained concrete after the freeze-thaw damage, fatigue tensile tests with four different loading paths were conducted on air-entrained concrete after 0, 100, 200 and 400 freeze-thaw cycles. The four different loading paths contained the monotonic (M) test where the envelope stress-strain curve was obtained, the cycles with constant strain increment (CSI) test where the variation of elastic modulus on the whole stress-strain curve was studied, the cycles to variable maximum strain amplitude (VMS) test where the low-cycle fatigue behaviour at different strain levels was analysed and the cycles with CMS' test, which was designed to analyse the post-peak behaviour of the specimens. Experimental results indicated that the properties of the air-entrained concrete basically remained unchanged under 200 freeze-thaw cycles, including the mass loss rate, tensile strength, elastic modulus and the dissipated energy per unit volume. While the freeze-thaw cycles increased over the critical value, the energy resulted from the cyclic load was not released from the materials and accumulated inside the materials fast. Energy accumulation directly led to the deterioration of the air-entrained concrete. To observe the pore structure of the air-entrained concrete, the scanning electron microscope test (SEM) was also adopted in this paper.
\end{abstract}

Keywords. Low-cycle fatigue; air-entrained concrete; freeze-thaw cycles; post-peak behaviour; scanning electron microscope (SEM).

\section{Introduction}

Concrete structure in service is often subjected to the freeze-thaw damage. Affected by the freeze-thaw cycles (FTCs), concrete deterioration takes place even if the load is far less than its strength. To deal with the frost damage, air-entrained concrete has been wildly used. Previous studies show that the freeze-thaw damage leads to the obvious reduction of strength and elastic modulus of concrete under the static load [1-3]. Under fatigue load, Hasan et al [4] have conducted the compression test and discussed mechanical properties of frost damage concrete. At present, most of the related studies are based on compression test. In order to popularize the application of air-entrained concrete better, the properties of air-entrained concrete in tensile fatigue need to be studied more thoroughly.

The typical freeze-thaw damage contains two parts: surface scaling and microstructure damage. The microstructure damage includes the irreversible tensile deformation and randomly oriented microcracks [5]. Lowstrain cyclic compressive load compacts the microcracks and enhances the concrete capacity [6]. The current

*For correspondence

Published online: 26 July 2019 research works are mainly concerned about the compressive test. Models have been established to simulate the compressive stress-strain curves of various concretes after the FTC [7-13]. Compared with the compressive mechanical properties, the irreversible tensile deformation probably has greater impacts on mechanical properties. The tensile behaviours are the key point for concrete calculation on crack width and structure elastic modulus in practical projects.

Since 1988, air-entraining agents have been widely used in the frost-resistance concrete [14]. It is well known that the air-entraining agent is usually used to improve the durability of concrete [15]. For this fact, it is necessary to study the tensile mechanical properties of concrete with the air-entraining agent after freeze-thaw damage.

With the development of mechanical properties analysis, a series of research works have been carried out on the whole curve and the softening process after the stress peak. Sinha et al [16] have studied the stress-strain relationship of concrete under cyclic compressive loading. An analytical model for the stress-strain relationship of concrete was presented. In the research, the monotonic loading was used to determine the stress-strain loading curve of concrete, and the concept on the uniqueness of envelope curve was 
proposed. Therefore, in this paper, the monotonic loading (M) was applied to determine the envelope curve. In Sinha's study, the cycles with constant strain increment (CSI) loading path were used for the first time to obtain the compressive elastic modulus of concrete under different stages of destruction. Maekawa and co-workers [17-19] introduced the elastic-plastic model into the simulation of the strain-softening stage, and the fatigue compression tests under strain control were carried out. The study shows that concrete has stress relaxation. When the total deformation is constant, the stress inside the concrete decreases slowly with time. Freeze-thaw damage is a kind of deterioration phenomenon, and the effect of frost damage on elastoplastic material is not clear. In this paper, combined with the CSI loading condition of Sinha and the strain-controlled cyclic loading method of Maekawa, the variable maximum strain amplitude (VMS) loading path is proposed for the analysis on the elastoplastic characteristics and strain relaxation under different stages of destruction. The effect of FTC on the elastoplastic characteristics of materials is analysed. The energy theory is introduced to the analysis on results of the cycles with CMS', which is the post-peak cyclic loading controlled by force. The hysteresis loop and the material dissipation energy difference caused by freezethaw damage are studied qualitatively.

In this paper, the tensile cycle tests were conducted on air-entrained concrete with different FTCs. Based on the previous studies, the strain control mode and the stress control mode were both accepted in the cyclic loading test $[20,21]$. Four loading methods are designed to obtain the elastic modulus and deformation of concrete through the stress-strain curve and to study the effects of freeze-thaw damage on the elastoplastic characteristics and dissipation energy of concrete materials. Referring to the previous research [6] on the compressive mechanical properties of concrete after freeze-thaw cycling, qualitative comparison has been conducted between the tensile behaviour and compressive behaviour after freeze-thaw cycling in the aspects of failure mode, the peak value variation and the whole curve.

\section{Experimental program}

\subsection{Materials}

The ordinary Portland cement with strength grade 42.5 MPa, river sand, the coarse aggregate with a maximum grain size of $15 \mathrm{~mm}$, tap water and high efficiency carboxylic acid water reducing agent were used in the sample. The concrete mixture property of cement, water, sand, aggregate, water reducer and air entraining agent is 410 : 201: 694: 1132: 2.05: 0.0205 . The unit is $\mathrm{kg} / \mathrm{m}^{3}$. The air content of the specimens is $3.9 \%$. Cylindrical polyether chloride (PVC) tubes with the size of $110 \mathrm{~mm}$ inner diameter and $280 \mathrm{~mm}$ height were used as moulds. A vibration table was employed to ensure the quality of casting after pouring. All the specimens were demoulded 1 day after casting, and then taken for outdoor sprinkler curing for 28 days.

\subsection{FTCs test}

Before FTCs test, the test specimens were fully saturated in $20 \pm 2^{\circ} \mathrm{C}$ water for 4 days. The quick FTCs test is referred to as 'the test method on long term and durability of concrete' GB/T50082-2009 (China). A freeze-thaw testing machine (HDK) was used in this test. The specimens were divided into four groups, corresponding to $0,100,200$ and 400 FTCs. The number in specimens' name represents the number of the FTCs. For example, the specimen F100 is the concrete specimen subjected to 100 FTCs.

When the predetermined FTCs were reached, the temperature of the testing machine recovered to room temperature slowly; at this stage the specimens were picked out and external damage inspection was conducted. The saturated mass $W_{n}$ of specimens was weighed.

The frost damage caused surface spalling. With the numbers of the FTCs increasing, the quality of the specimens got worse. As figure 1 shows, after freeze-thaw test, the surface concrete began to peel off and the surface scaling resulted in aggregation exposure.

\subsection{Loading path}

To study the variation of elastic modulus, the fatigue behaviour under different strain levels and the post-peak softening curve of the concrete after different FTCs, four loading paths were designed in the tests, including the monotonic (M) test, where the envelope stress-strain curve was obtained, the cycles with CSI test, where the variation of elastic modulus was studied, the cycles to VMS test, where the low-cycle fatigue behaviour at different strain levels was analysed, and the cycles with CMS' test, which was mainly focused on the fatigue behaviour of the

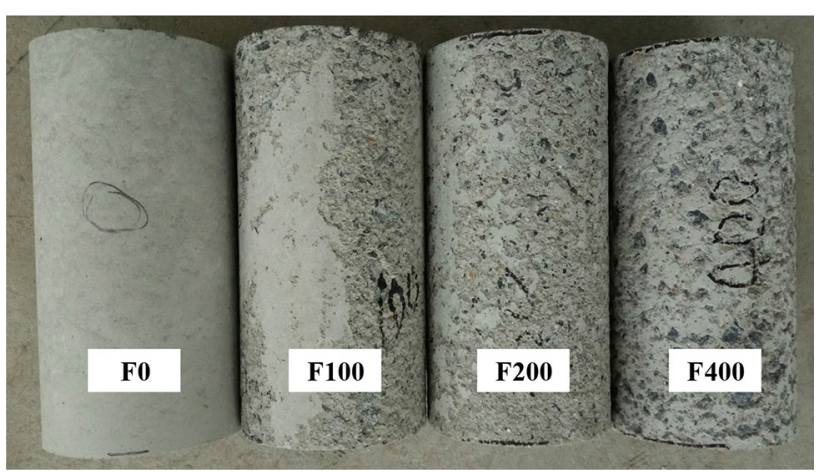

Figure 1. The samples of group F0, F100, F200 and F400. 


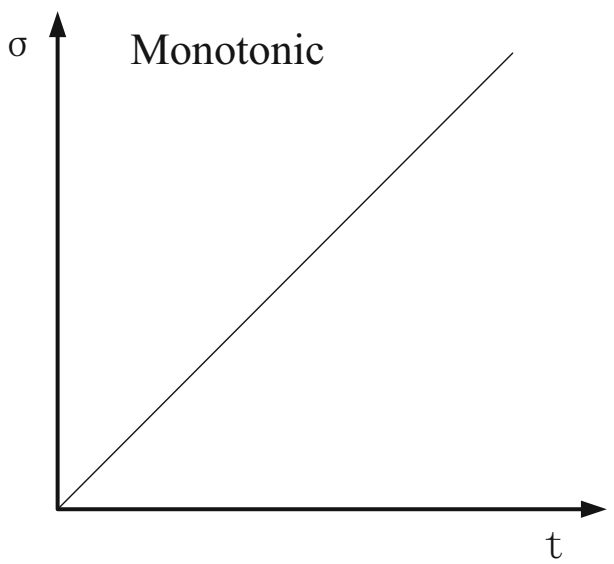

(a)

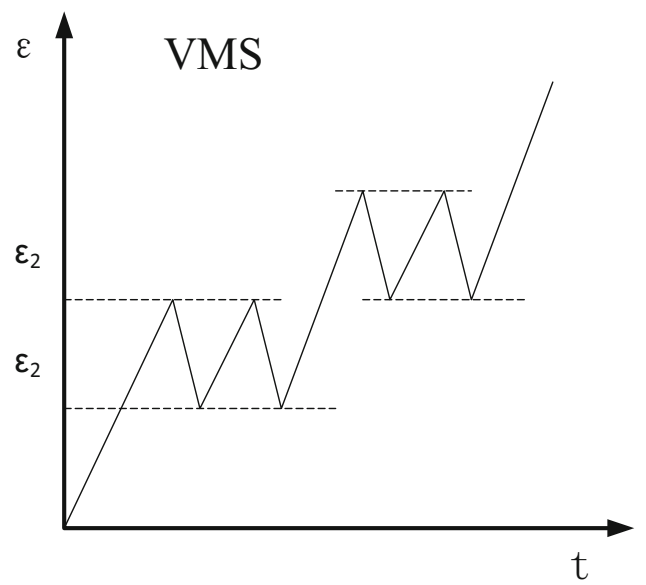

(c)

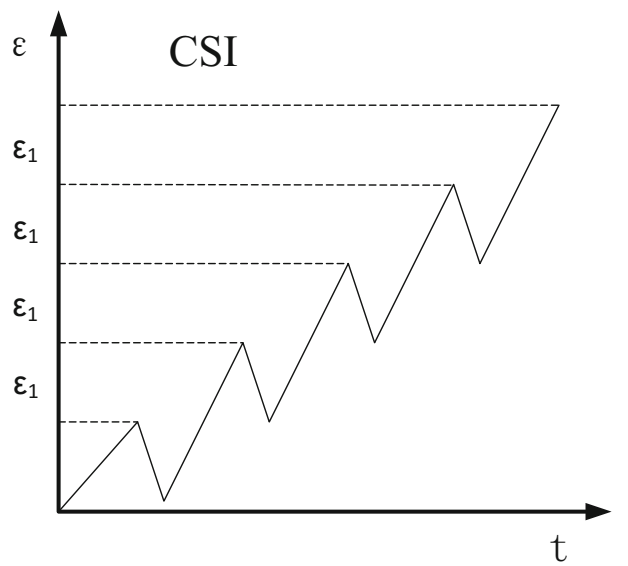

(b)

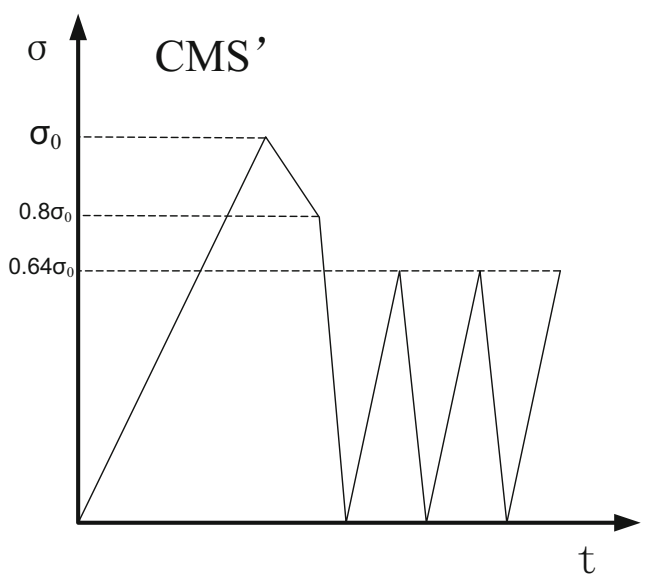

(d)

Figure 2. The diagrams of loading paths.

specimens after the peak. The diagrams of the loading paths are shown in figure 2. The accurate loading paths are shown as follows:

(a) Monotonic test (M).

(b) Cycles with CSI. After loading to the target strain, stress unloads to 0 , then reloads again to the next target strain.

(c) Cycles to VMS. There are five loading levels. After loading to the target strain, stress unloads to 0 , then reloads to the target strain. These actions are repeated 14 times in each loading level.

(d) Cycles with CMS'. Previous loading paths are mainly about strain control methods, while the 4th loading path adopts the stress control. Based on the previous studies, the post-peak unloading stress of specimens is controlled at 0.64 times the peak stress [22, 23].

To obtain a proper number of loading and unloading processes that can effectively reflect the mechanical properties variation during the concrete destruction in the CSI and VMS test, the intervals $\left(\varepsilon_{1}\right.$ and $\left.\varepsilon_{2}\right)$ of unloading strain are not constant. The test includes pre-peak stage and post-peak stage. Due to the dispersion of peak stress, it is impossible to distinguish the pre-peak and post-peak stages accurately. In order to reflect the elastic modulus variation better, the interval of unloading strain around the peak value needs to be adjusted. The relationships between strain and time are shown in the figure 3. Meanwhile, the symbol in specimens' name represents the type of the loading path.

\subsection{Cyclic loading test}

Considering the uneven temperature distribution in both ends of specimens during curing time, before the cyclic loading test, the specimens were cut into $200 \mathrm{~mm}$ height cylindrical specimens with a diameter of $110 \mathrm{~mm}$. Each end of the specimens was pasted by a steel plate with the same 


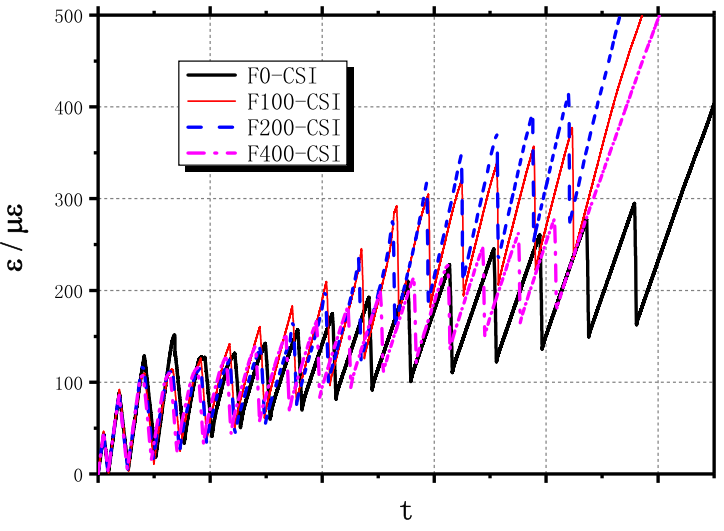

(a)

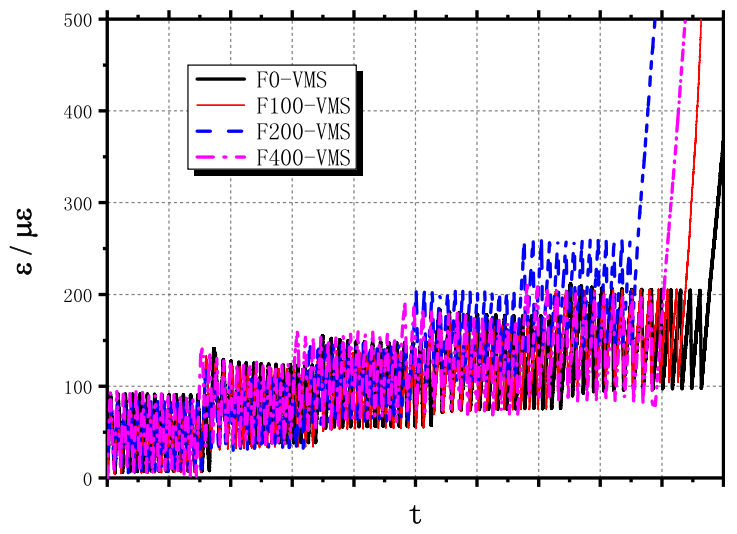

(b)

Figure 3. The strain-time curve: (a) CSI and (b) VMS.

diameter. To ensure firm bond between specimens and the testing machine, a structure adhesive with $10 \mathrm{MPa}$ tensile strength was utilized as the bonding mastic.

A closed loop electro-hydraulic servo testing machine MTS322 was adopted in these tests. Three LVDTs (linear variable differential transducer) were distributed across the lateral surface of the specimen. The measured data of the load and deformation were used as the feedback signal to control the load process.

\subsection{Scanning electron microscope test (SEM)}

A scanning electron microscope (SEM) Hitachi S-4800 model with $20.0 \mathrm{kN}$ acceleration voltage was adopted in this work. The samples of freeze-thaw damage concrete were cut at $25 \mathrm{~mm}$ from the surface of the specimen with 3 $\mathrm{mm}$ thickness, $2 \mathrm{~mm}$ width and $2 \mathrm{~mm}$ length. The sample was maintained in anhydrous ethanol before the hydration reaction was completely over. Before the SEM test, all the samples had been baked in an oven under $50{ }^{\circ} \mathrm{C}$. The natural section without polishing was selected as the test section.

\section{Effect of freeze-thaw damage}

The mass loss rate, relative elastic modulus and the SEM figures affected by the freeze-thaw cycling are shown in this section.

\subsection{Mass loss rate after freeze-thaw test}

The mass loss rate of concrete specimens after freeze-thaw cycling was calculated as follows:

$$
\Delta W=\frac{W_{0}-W_{n}}{W_{0}} \times 100 \%
$$

where $\Delta W$ is the concrete mass loss rate after $n$ FTCs; $W_{0}$ is the concrete mass before the FTC test and $W_{n}$ is the concrete mass after $n$ FTCs.

In this work, F0, F100, F200 and F400 four groups of specimens were weighed; the mass data is shown in figure 4. According to GB/T50082-2009(China), the freezethaw label of concrete is defined as the maximum number of FTCs corresponding to the maximum mass loss rate of the specimen not exceeding 5\% and the relative elastic modulus value not less than $60 \%$. According to figure 4 , the maximum mass loss rate after 400 FTCs is $0.51 \%$, which is much lower than the limit. It proves that concrete with air

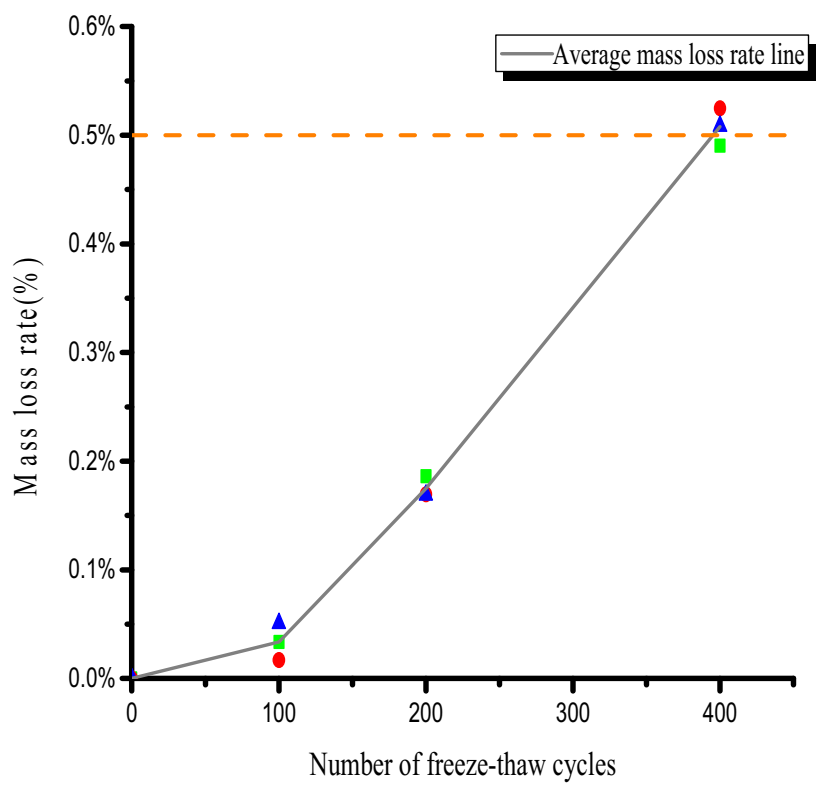

Figure 4. The curve of the mass loss rate vs the FTC. 
entraining agent has excellent frost resistance behaviours. Under 100 FTCs test, the concrete mass basically remained unchanged, while the mass loss rate increased after 100 times.

\subsection{Dynamic elastic modulus loss after freeze- thaw}

The freeze-thaw damage is a kind of physical process that results in material softening. The internal cracks development and the hole space increase have significant influence on the dynamic elastic modulus of the concrete. Powers [24] put forward the theory of hydrostatic pressure and osmotic pressure theory to explain the freeze-thaw damage on the concrete. In the theory, when suffering from high and low temperature alternation, the pore structure of the concrete will be subjected to fatigue stress consisting of frost heave pressure and seepage pressure. This force will lead to the erosion on concrete surface and the damage in microstructure. As the number of FTCs increases, the damage gradually accumulates.

A test machine NM-4B non-metallic ultrasonic testing analyser was applied to test the velocity of P-wave and verify the afore-mentioned theories. On account of the following equation, the dynamic elastic modulus can be obtained [25]:

$$
E_{d}=\frac{(1+\mu)(1-2 \mu)}{(1-\mu)} \rho v^{2}
$$

where $E_{d}$ is the dynamic elastic modulus of specimens and $\mu$ is the Poisson ratio; the corresponding value of concrete usually adopted is $0.15 ; \rho$ is the density of specimens and $v$ is the $\mathrm{P}$-wave velocity.

The relative dynamic elastic modulus is calculated as follows:

$$
P_{n}=\frac{E_{n}}{E_{0}}
$$

where $P_{n}$ is the relative dynamic elastic modulus after $n$ FTCs; $E_{n}$ is the dynamic elastic modulus and $E_{0}$ is the dynamic elastic modulus before the test. The units of $E_{n}$ and $E_{0}$ are $\mathrm{MPa}$. The relative dynamic elastic modulus $P_{n}$ is the mean of three specimens test data and is used to evaluate the dynamic elastic modulus loss. Figure 5 shows the test data in the process from 0 to 400 cycles. Every 50 cycles, three specimens were picked out and tested.

As illustrated in figure 5, the value of the relative dynamic elastic modulus decreases with FTCs. As shown in Eq. (2), the dynamic elastic modulus is determined by the test velocity of P-wave. Based on the law of wave propagation, owing to the increase in the number and size of microcracks, the medium in the wave transmission path changes and the velocity of P-wave is reduced. Hence, the changing of wave velocity and dynamic elastic modulus

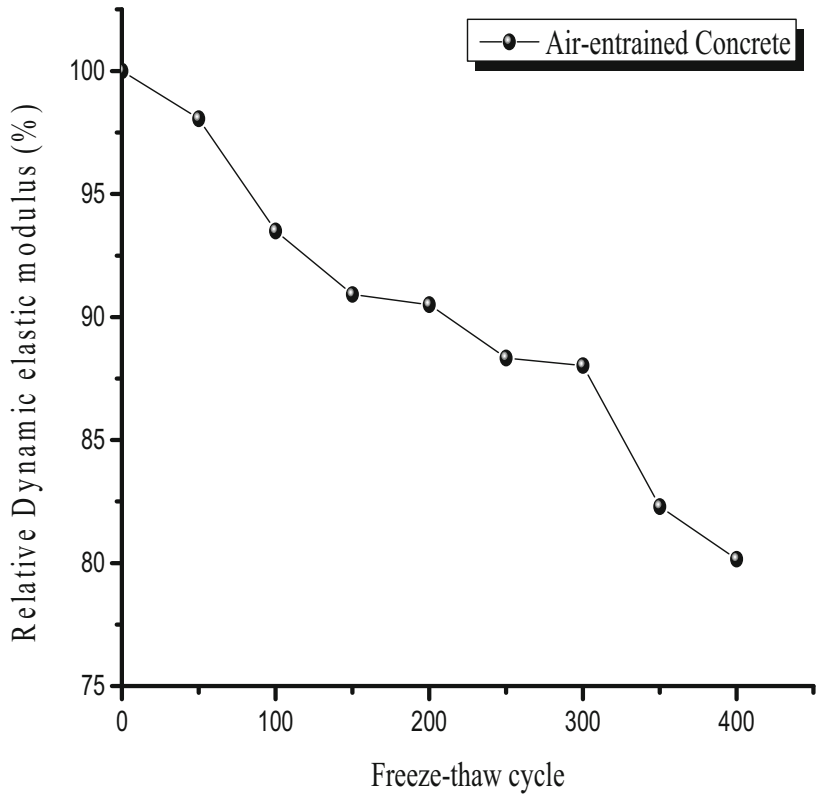

Figure 5. The curve of the relative elastic modulus vs the FTC.

confirms the fact that the microcracks gradually accumulate for the effect of FTC.

The curve of the relative dynamic elastic modulus presents three stages. Under 150 FTCs, the relative dynamic elastic modulus decreases rapidly. Considering the hydrostatic pressure influence, in this stage, a large number of microcracks are generated around the pores and expand the pore volume to fit the water phase change. From 150 FTCs to $300 \mathrm{FTCs}$, the values of the relative dynamic elastic modulus become stable. The reason is that the formation of microcracks in the first stage weakens the influence of frost heave pressure. The opening and closing process of microcrack improved the behaviour of the concrete on adjusting the volume change. In this stage, the number of microcracks was basically unchanged. In the last stage, from 300 FTCs to 400 FTCs, the elastic deformation of microcracks cannot sustain the accumulated pore water volume. The microcrack got larger. The larger cracks accelerated the progress of pore water accumulation. As a result, the frost heave pressure increased, which, ultimately, led to the great jump of the dynamic elastic modulus.

In comparison, the minimum relative dynamic elastic modulus of the air-entrained concrete is over $80 \%$, much better than that of ordinary concrete.

\subsection{Microstructure change after the FTC test}

Figure 6 shows the comparison between the ordinary concrete and the air-entrained concrete. There are more cracks in air-entrained concrete seen under the electron microscope, which may be due to the physical damage during the 

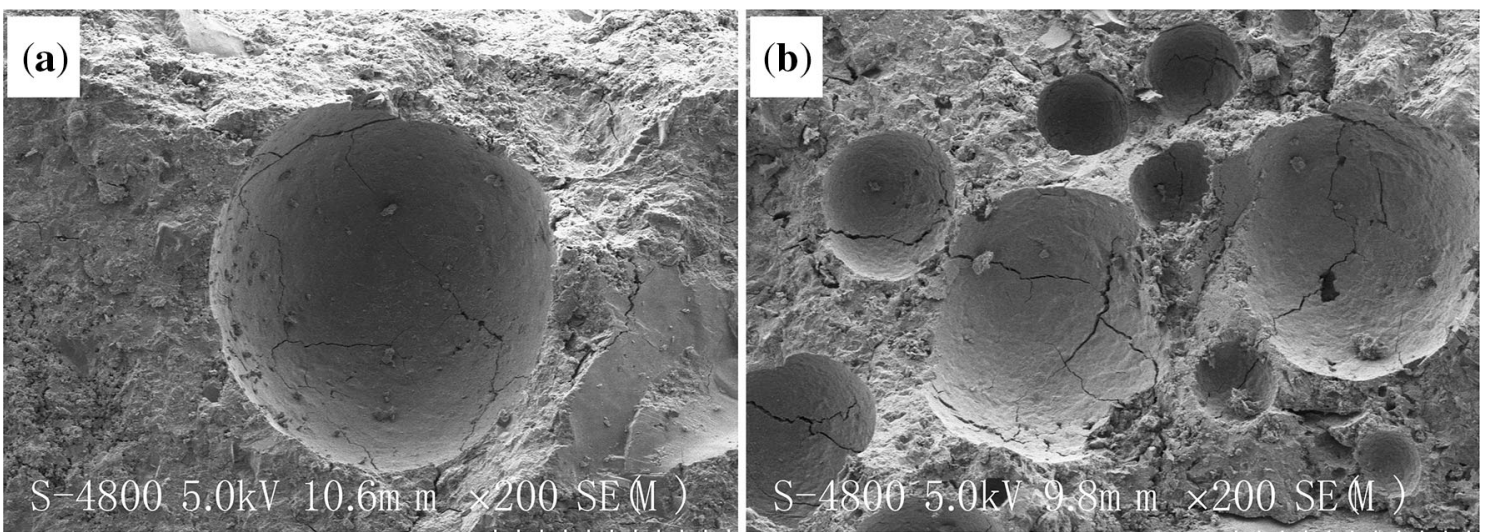

Figure 6. The specimens before the freeze-thaw test magnified 200 times: (a) ordinary concrete and (b) air-entrained concrete.

sampling. During the curing time, cracks are formed inside the concrete. They can be divided into connected cracks and disconnected cracks. It is generally believed that, under freeze-thaw conditions, the cracks connected to the outside are more harmful. Most of the cracks in the figures were located around the pores and did not connect with the outside. Based on Powers' hydrostatic pressure theory [24], with the decrease of temperature and the increasing of ice volume, the unfrozen pore solution continues to be compressed. During the migration of solution inside the pore, the viscous resistance must be overcome. The longer the moving distance, the greater the viscous resistance that needs to be overcome. The hydrostatic pressure increases, which causes the crack. As figure $6 \mathrm{~b}$ shows, the concrete with air-entrained agents has more small bubbles to optimize the gradation of bubbles and shorten the distance between bubbles. This pore structure of air-entrained concrete has better behaviour when subjected to stress [26].

In figure 7, the samples of group F0, F100, F200 and F400 have been scanned. The pore structure of group F0 is integrated. From group F100 to group F400, the microcracks got deeper and deeper. Individually speaking,
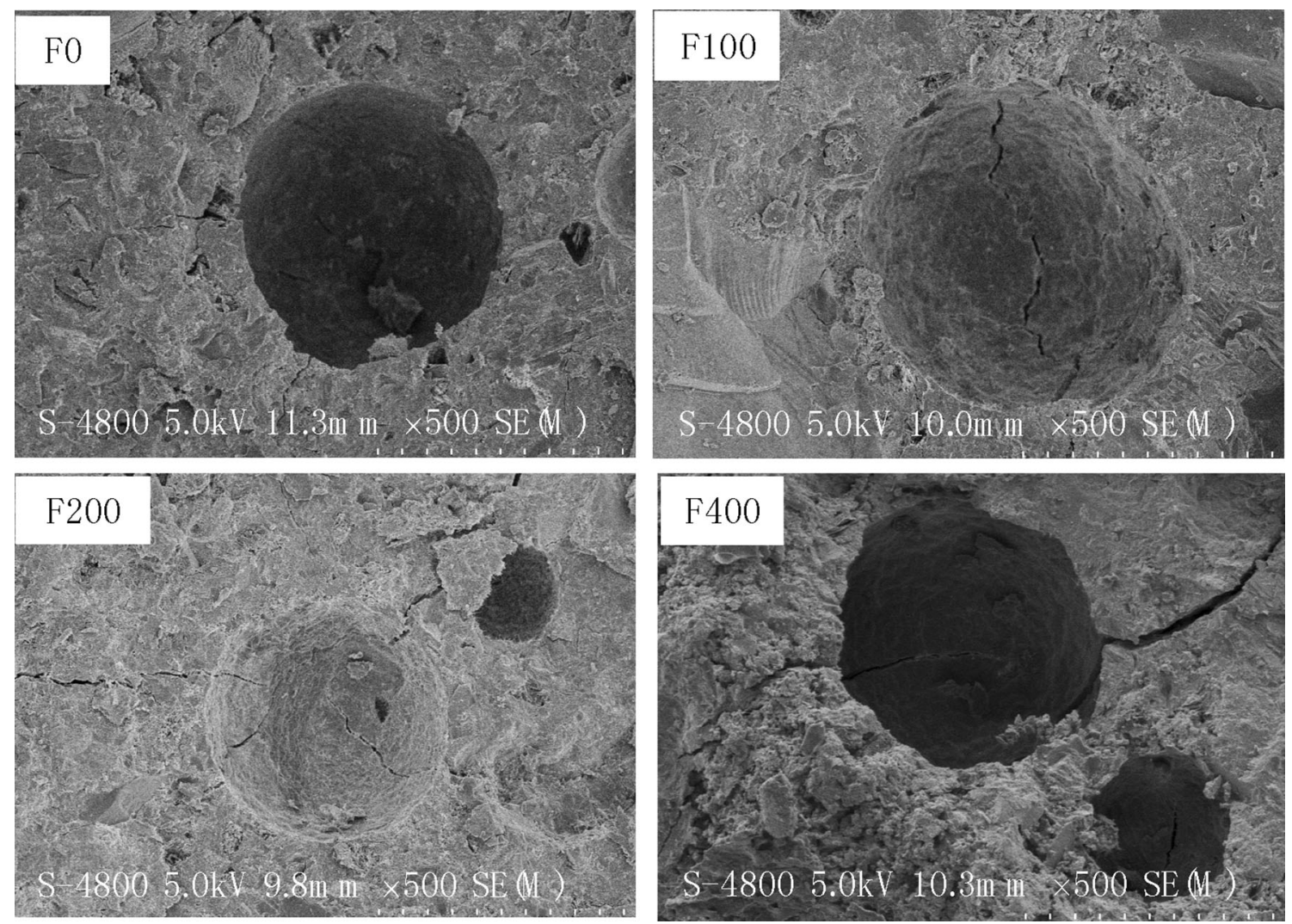

Figure 7. The micro-change under the freeze-thaw cycle test magnified 500 times. 

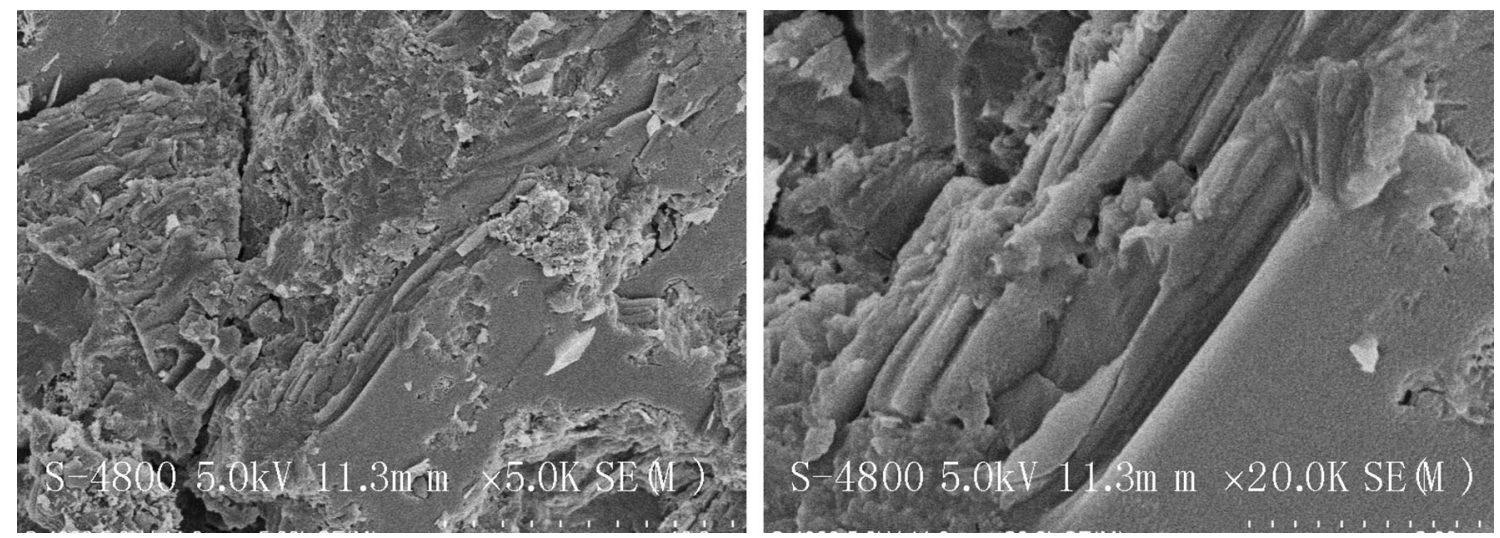

Figure 8. The SEM diagrams of group F0 magnified 5k and 20k times.

microcracks in group F100 are small and shallow. In group F200, microcracks were more; some of them became longer but were still shallow. In group F400, one crack went through the bubble and had a deeper and longer shape. Meanwhile, compared with the first three groups, the material around the bubble softened in group F400. The edge of bubble became rough and the number of microcracks increased. After pore water freezing, the surface of pore structure was subjected to the force resulted from the water volume expansion; then microcracks appeared. With the cycles increasing, microcracks grow, and then result in the microstructure damage. As figure 7 shows, the microstructure variation in the SEM diagrams proves the explanation on the three stages changing of the relative dynamic elastic modulus.

Figures $8,9,10,11$ are the SEM diagrams of air-entrained concrete magnified $5 \mathrm{k}$ times and $20 \mathrm{k}$ times. The initial microstructure of the samples is completed. C-S-H gel knitted and grew with the crystal $\mathrm{Ca}(\mathrm{OH})_{2}$. There is evidently no weakness found in the microstructure. After 100 cycles, six-diamond small flake crystal $\mathrm{Ca}(\mathrm{OH})_{2}$ began to separate out while a few needle-shaped ettringites appeared. The cracks between $\mathrm{C}-\mathrm{S}-\mathrm{H}$ gel and $\mathrm{Ca}(\mathrm{OH})_{2}$ appeared. After 200 cycles, from 20k times diagram, it is clear that the number of the flake crystals and the ettringites increased, but the texture of concrete material basically remained as in the 5k times figure. After 400 cycles, the ettringites filled up the cracks and enlarged the crack space. After 400 cycles, it was found that a large number of needle-like ettringites grew between pores and cracks, enlarged the crack space and covered the cracks at low magnification (5k times). At high magnification (20k times), compared with figure 10, the needle crystals increased obviously, ettringites were precipitated and the bedded structure was seriously destroyed. With ettringites growth, the microstructure and chemical composition of concrete changed. The frost resistance of air-entrained concrete gradually disappeared.

\section{Cyclic tensile properties of frost damage concrete}

\subsection{Failure mode}

The fracture position was randomly distributed in the concrete specimens, while the adhesive plane between the steel plate and specimen was hard enough to transfer the tensile load to the concrete specimens.
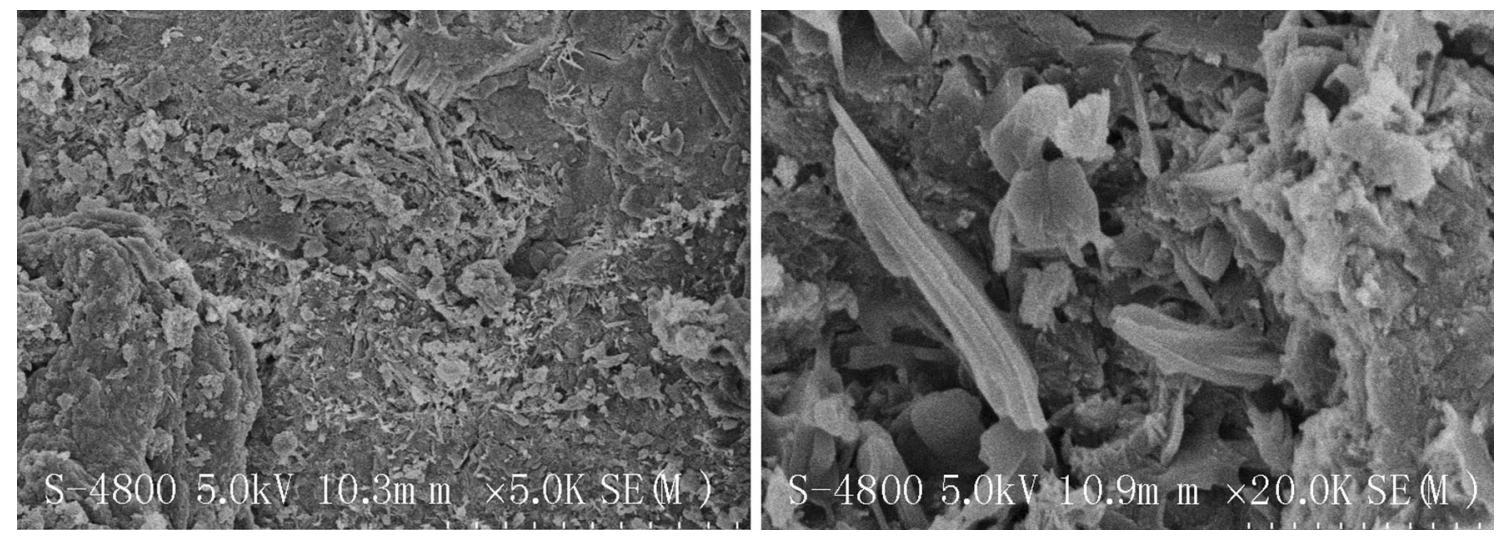

Figure 9. The SEM diagrams of group F100 magnified 5k and 20k times. 

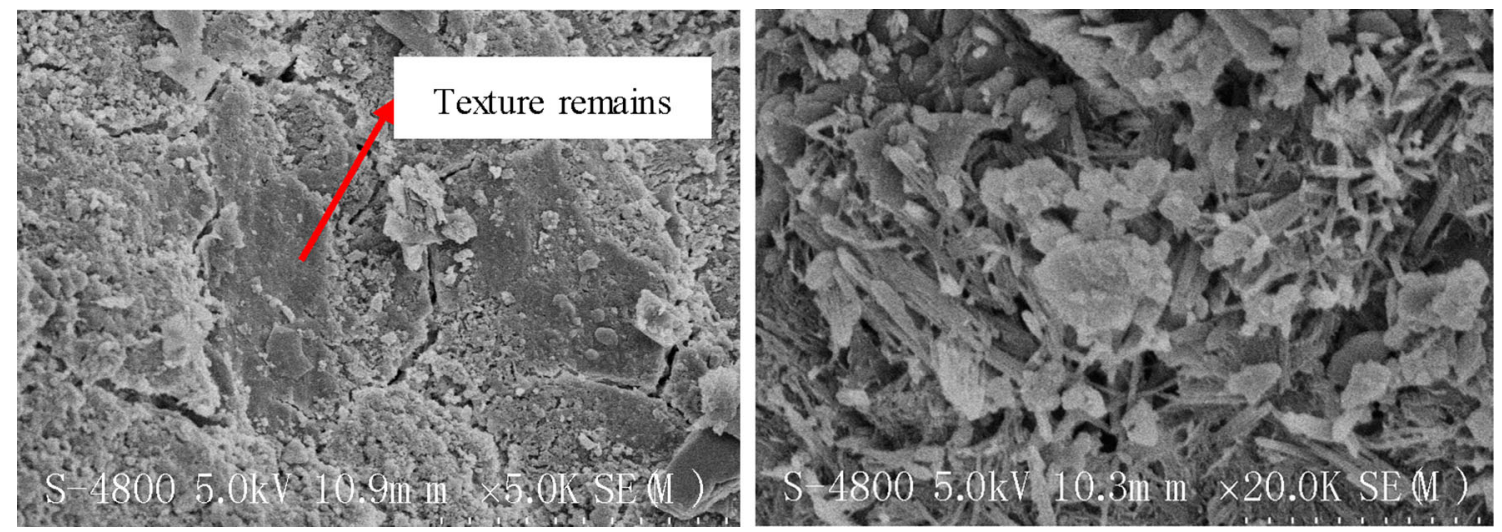

Figure 10. The SEM diagrams of group F200 magnified 5k and 20k times.
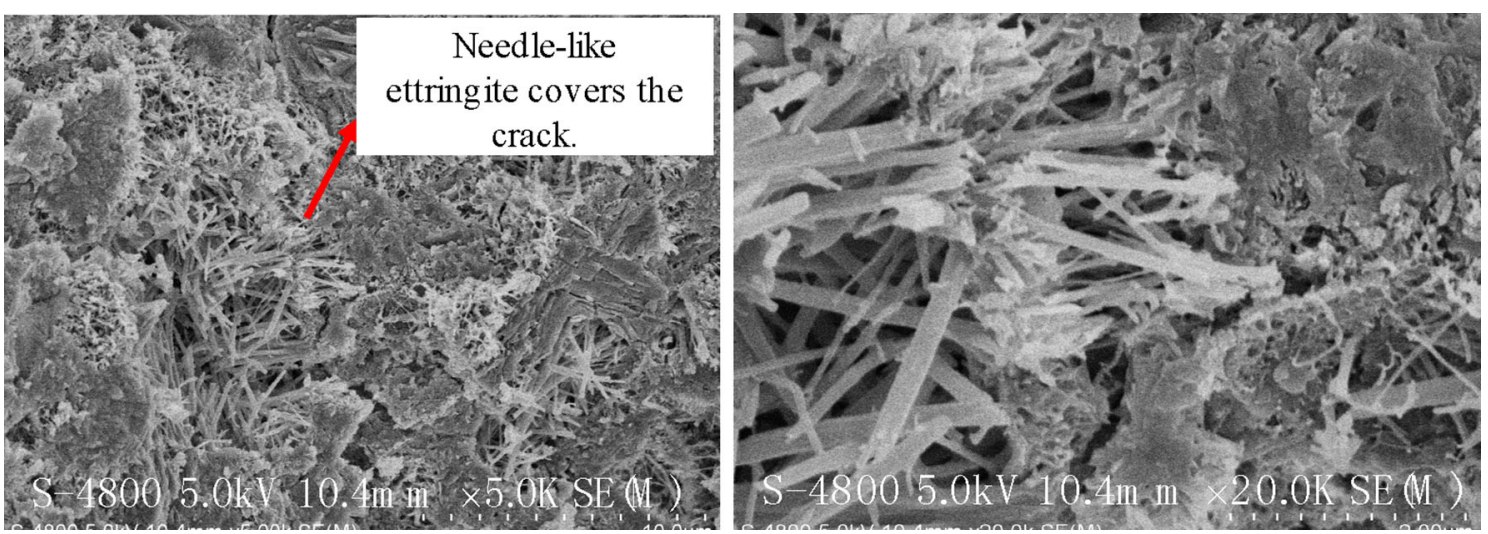

Figure 11. The SEM diagrams of group F400 magnified 5k and 20k times.

\subsection{Mechanical properties before the peak}

Table 1 shows the average peak strain, stress and initial elastic modulus of the specimens under different FTCs and different loading paths. The group number including the letter ' $\mathrm{XU}$ ' is the reference data, which is introduced to compare the tensile properties vs the compressive ones [6]. The values of the peak stress and the peak strain are discrete. Concrete is a kind of quasi-brittle material, which directly causes the high discreteness of the test data. In summary, with increasing FTCs, the peak stress tended to decrease.

Each datum in the table was calculated from the values of three effective specimens in the same group, including the average peak strain $\bar{\varepsilon}$, the coefficient of variation of the peak strain $c_{v}(\varepsilon)$, the average peak stress $\bar{\sigma}$, the coefficient of variation of the peak stress $c_{v}(\sigma)$, the initial elastic modulus $E_{40}$ and the coefficient of variation of elastic modulus $c_{v}(E)$. The initial elastic modulus is defined as the tangent elastic modulus corresponding to 40 percent of the peak stress. To compare the parameters of each group, the probability distribution variation of the parameters is normalized, and the coefficient of variation is calculated as follows:
Table 1. The main mechanical properties of concrete under cyclic loading.

\begin{tabular}{lcccrcc}
\hline $\begin{array}{l}\text { Group } \\
\text { number }\end{array}$ & $\bar{\varepsilon}$ & $\begin{array}{c}c_{v}(\varepsilon) \\
(\%)\end{array}$ & $\begin{array}{c}\bar{\sigma} \\
(\mathrm{MPa})\end{array}$ & $\begin{array}{c}c_{v}(\sigma) \\
(\%)\end{array}$ & $\begin{array}{c}E_{40}\left(10^{4}\right. \\
\mathrm{MPa})\end{array}$ & $\begin{array}{c}c_{v}(E) \\
(\%)\end{array}$ \\
\hline F0-M & 127.77 & 2.31 & 3.97 & 4.74 & 3.80 & 3.25 \\
F100-M & 96.60 & 0.72 & 3.27 & 4.41 & 3.91 & 5.53 \\
F200-M & 120.89 & 3.18 & 3.54 & 7.64 & 3.69 & 1.98 \\
F400-M & 106.44 & 3.32 & 2.78 & 9.86 & 3.33 & 2.11 \\
F0-CSI & 119.60 & 4.05 & 3.57 & 12.84 & 3.71 & 3.16 \\
F100-CSI & 106.33 & 0.58 & 3.53 & 4.99 & 3.78 & 2.32 \\
F200-CSI & 122.48 & 6.25 & 3.63 & 5.83 & 3.69 & 2.78 \\
F400-CSI & 117.64 & 10.05 & 3.00 & 7.51 & 3.29 & 5.52 \\
F0-VMS & 147.69 & 10.45 & 3.86 & 13.58 & 3.80 & 1.54 \\
F100- & 125.06 & 12.59 & 4.05 & 5.24 & 3.84 & 5.55 \\
$\quad$ VMS & & & & & & \\
F200- & 123.90 & 4.17 & 3.62 & 5.91 & 3.66 & 4.70 \\
$\quad$ VMS & & & & & & \\
F400- & 136.91 & 9.45 & 3.41 & 16.38 & 3.27 & 6.63 \\
$\quad$ VMS & & & & & & \\
F0-XU & - & - & 41.21 & 6.4 & 3.533 & - \\
F50-XU & - & - & 29.12 & 21.9 & 0.247 & - \\
F100-XU & - & - & 21.58 & 22.0 & 0.077 & - \\
F150-XU & - & - & 16.91 & 27.8 & 0.028 & - \\
\hline
\end{tabular}




$$
c_{v}=(S D / M N) \times 100 \%
$$

where $c_{v}$ is the coefficient of variation, $S D$ is the standard deviation and $M N$ is the average value.

The change of initial elastic modulus is basically regular. With increasing FTCs, the value of the initial elastic modulus increases slightly and then declines. The assumption of local compaction proposed by $\mathrm{Xu}$, probably, is the reason for the slight rise [6]. The pore structure can be divided into two types: the open pores and the closed pores. Under the low FTCs, water cannot flow into the closed pore. Without water condensing, under the cold environment, the closed pore structure contracted and led to the local compaction. With increasing cycles, the microcracks joined the closed pore with the open pore. Affected by the osmotic pressure, water migrated from gel pore and accumulates in the closed pore structure. As the experimental data in table 1 illustrates, with the FTCs rising, the initial elastic modulus of the specimens decreased and the prepeak elastic properties of concrete declined. In the last four groups, the compressive strength reduces rapidly after frost damage. It is worth noting that there is a difference between the variation of $E_{40}$ affected by the FTCs and the result of figure 5. In figure 5, the dynamic elastic modulus keeps decreasing. Firstly, it is clear that both moduli reflect the elastic deformation capacity of the material. However, they are not the same in definition and calculation. The dynamic elastic modulus depends on the wave velocity. After frost damage, the micro-cracks formed, the medium in the wave transmission path changed and the wave velocity decreased significantly. $E_{40}$ is the slope value of the linear part in the stress-strain curve. Affected by the local compaction of micro-cracks, the bearing capacity appeared to be improved. As a result, the $E_{40}$ increased slightly. Dynamic elastic modulus is sensitive to microcracks. In the limited FTC, the effect of microcracks on mechanical properties of materials is not significant. In essence, different responses to micro-cracks caused the different variation.

Moreover, the specimens of the last four groups are ordinary concrete. Hence, after 50 cycles, the elastic modulus of the concrete drops down. After the frost damage, the concrete elastic properties are basically lost, while, in this paper, the tensile strength has a smaller decline.

\subsection{Elastic modulus variation}

Altering one specimen from each group as the sample, figure 12 shows the stress-strain curves of samples under the monotonic tensile test. According to the figure, the peak stress decreases with the FTCs. Before the peak, the elastic moduli of F0, F100 and F200 are basically equal. However, the elastic modulus of F400 is much lower than the others. According Xu et al [6], affected by the frost damage, the pre-peak stress-strain curve shows concave shape. As the FTCs increase, the concave shape becomes more and more

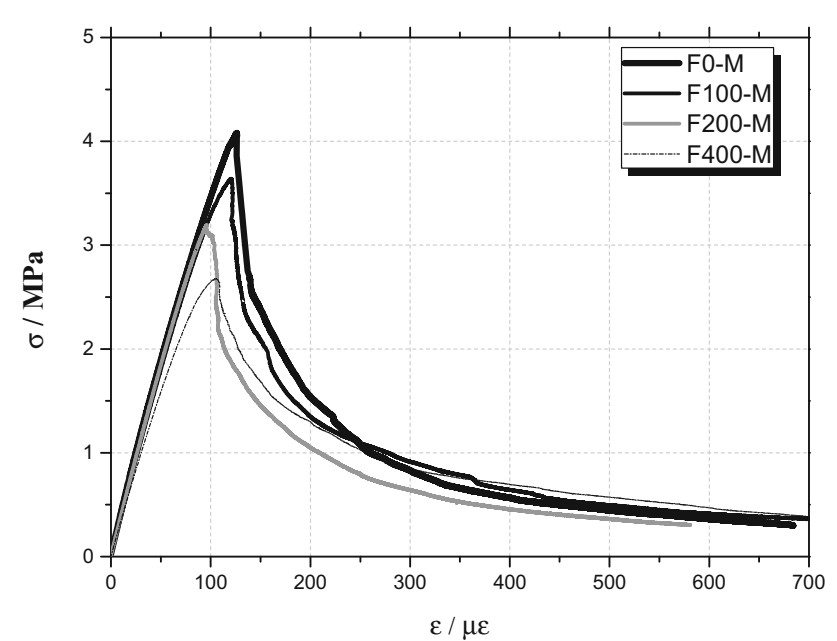

Figure 12. The stress-strain curves in monotonic tensile test.

obvious. The reason that $\mathrm{Xu}$ gives is that, after freezing, a large number of micro-cracks are generated, and, with the increase of the compressive stress, the compaction effect promoted the micro-crack closure, and then the tangent modulus increased. However, under the tensile condition, the compaction effect does not exist. Hence, the curve shapes before and after the frost damage are similar. In other words, it is more dangerous for concrete with frost damage subjected to tensile force than compressive force.

Figure 13 shows the stress-strain curves of samples in the CSI test. Before the peak stress, the curve in the CSI test is similar to the curve under the monotonic tensile test. After the peak stress, at the same strain level, the stress under the cyclic load is much lower than under the monotonic load. The stress-strain curve in the CSI test is always lower than the one in the monotonic test. The stress-strain curve in the monotonic load limits the range of the stress-strain curve under different cyclic loading paths, which is also fitted for the result of the VMS and CMS' tests.

Figure 14 shows the relations between the elastic modulus vs unloading strain under the CSI test. The post-peak elastic modulus of each cycle is defined as the slope between the unloading point and the reloading point in the stress-strain curve:

$$
E_{c s i}=\frac{\sigma_{e u}-\sigma_{l r}}{\varepsilon_{e u}-\varepsilon_{l r}}
$$

where $\sigma_{e u}$ is the unloading stress; $\varepsilon_{e u}$ is the unloading strain; $\sigma_{l r}$ is the reloading stress; $\varepsilon_{l r}$ is the reloading strain and $E_{c s i}$ is the elastic modulus of each cycle.

In figure 14, it is apparent that, with increasing FTCs, the initial elastic modulus declined and the final elastic modulus tended to be stable and converged to a certain value. The elastic modulus reduction presented three stages: the low-speed stage, the rapid stage and the final stable stage. 


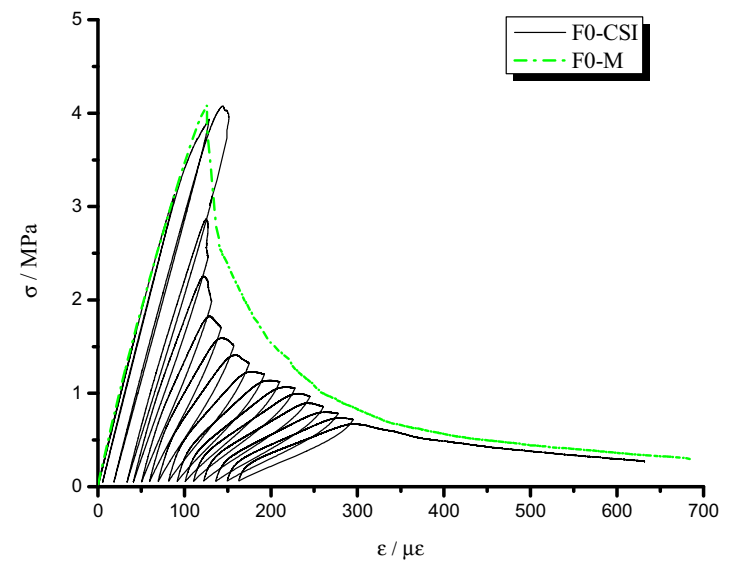

(a) F0

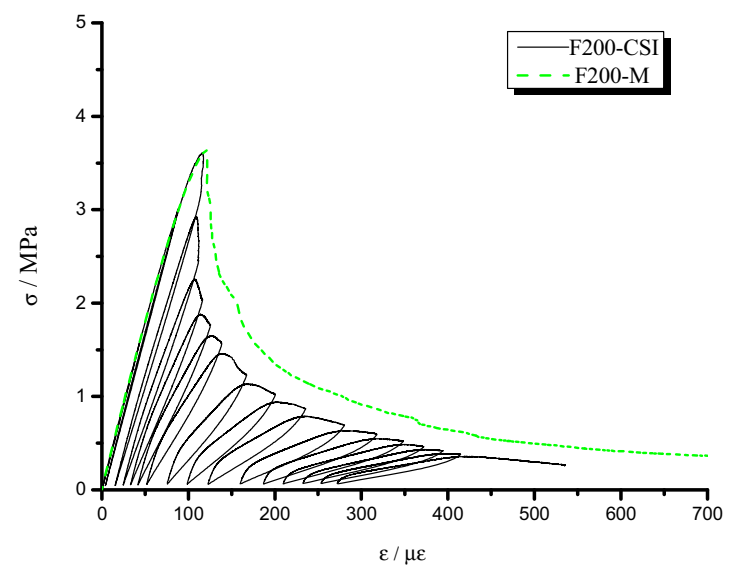

(c) F200

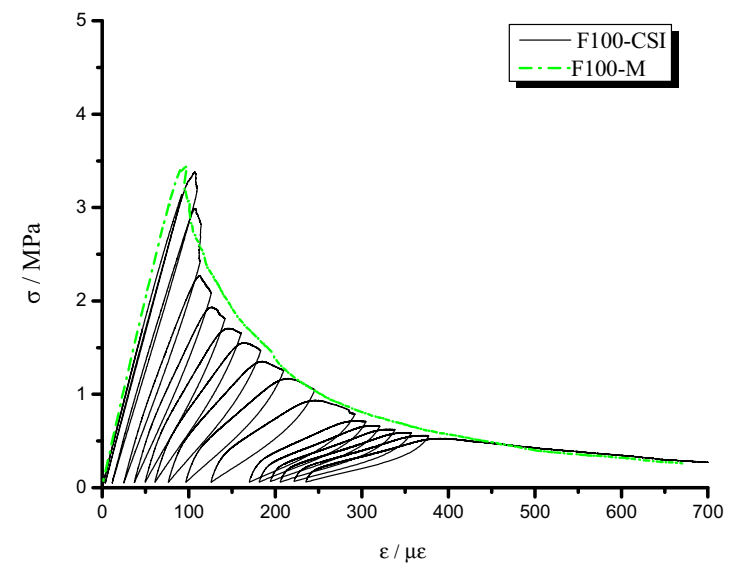

(b) F100

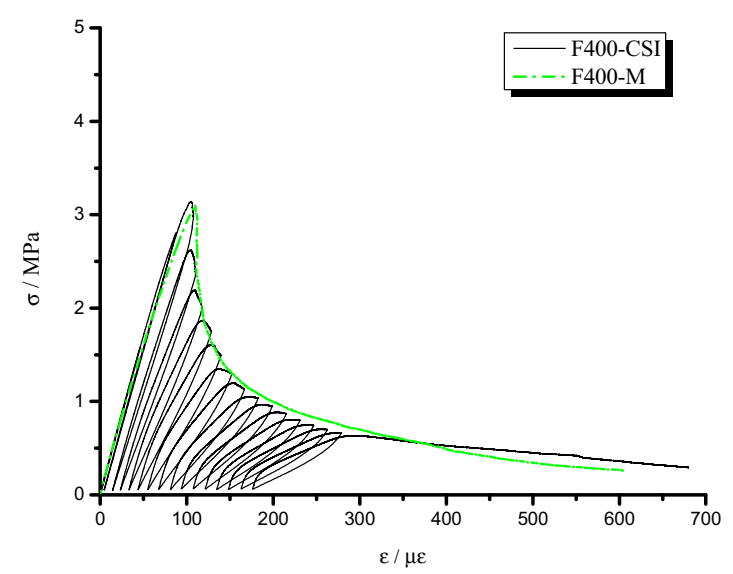

(d) F400

Figure 13. The stress-strain curves in the CSI test.

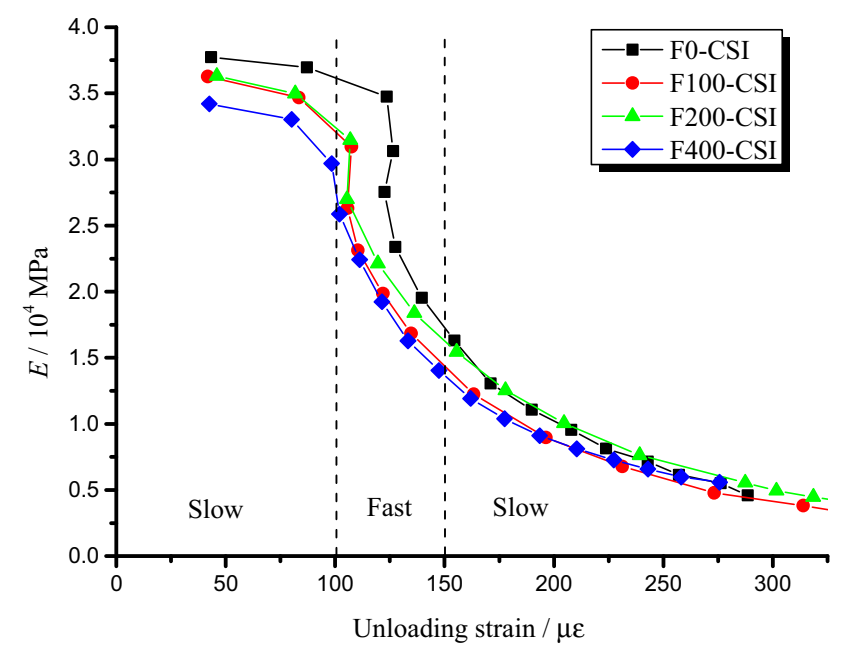

Figure 14. The curve of the elastic modulus vs the unloading strain in the CSI test.

The first stage corresponds to pre-peak stage. In this stage, the elastic modulus had a little decrease. Compared with unfrozen specimens, the elastic modulus of specimens after frost damage had larger reduction in the pre-peak process. With the unloading strain of the second stage ranging from $100 \mu \varepsilon$ to $150 \mu \varepsilon$, the elastic modulus decreased rapidly. The rapid development of the internal cracks in the post-peak process was the main reason for the decrease. In the last stage, the elastic modulus tended to be stable. At $250 \mu \varepsilon$, the elastic modulus was basically unchanged.

Figure 15 presents the stress-strain curve in the VMS test. Figure 16 shows the relations between the first ring elastic modulus and the unloading strain. The first ring elastic modulus is equal to the slope between the unloading point and reloading point of the first ring in the stress-strain curve. The variation of the first ring elastic modulus in the VMS test is basically consistent with the elastic modulus variation in the CSI test. The difference of curve shape in figures 14 and 16 resulted from the number of data points mainly. There are more data in figure 14, while the data in figure 16 are limited. As mentioned earlier, the elastic modulus of the material varies greatly around the peak stress and the number of data points in this range is especially increased in the CSI test. However, there are still something in common; especially, the pre-peak elastic 


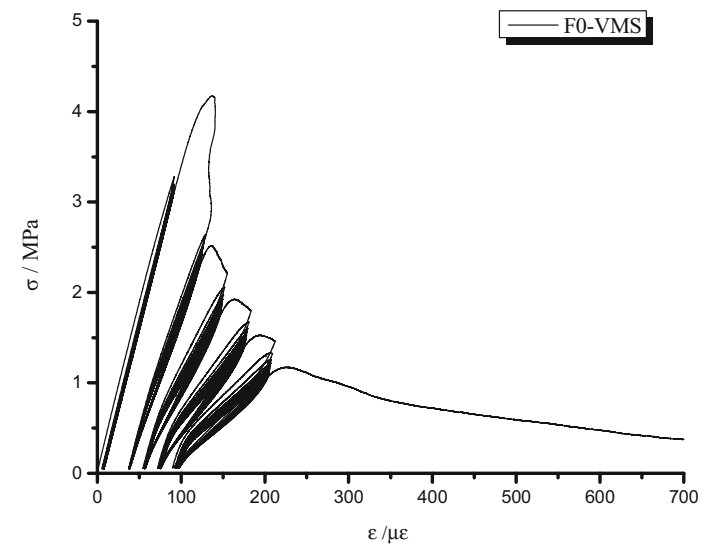

(a) F0

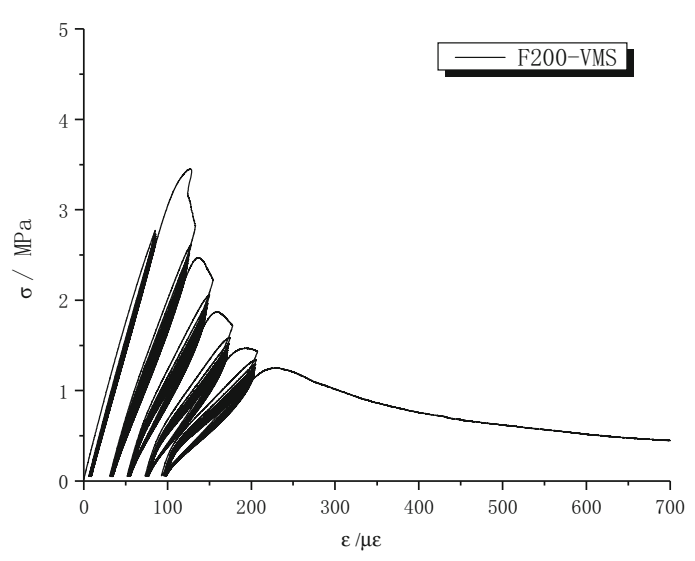

(c) F 200

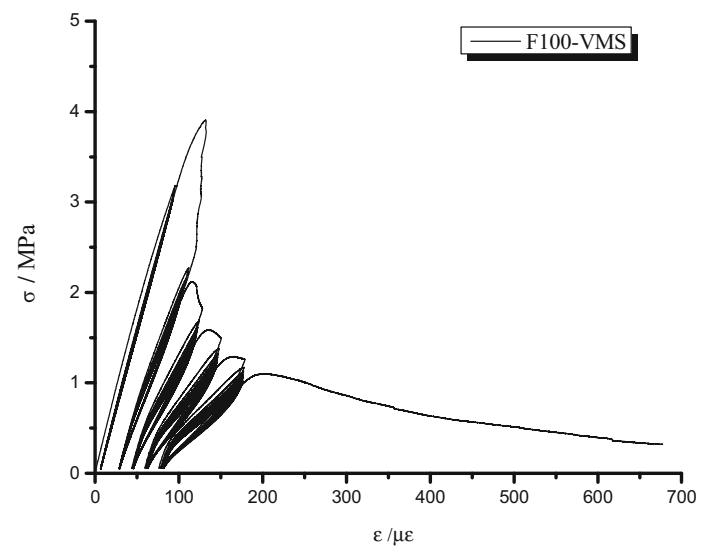

(b) F 100

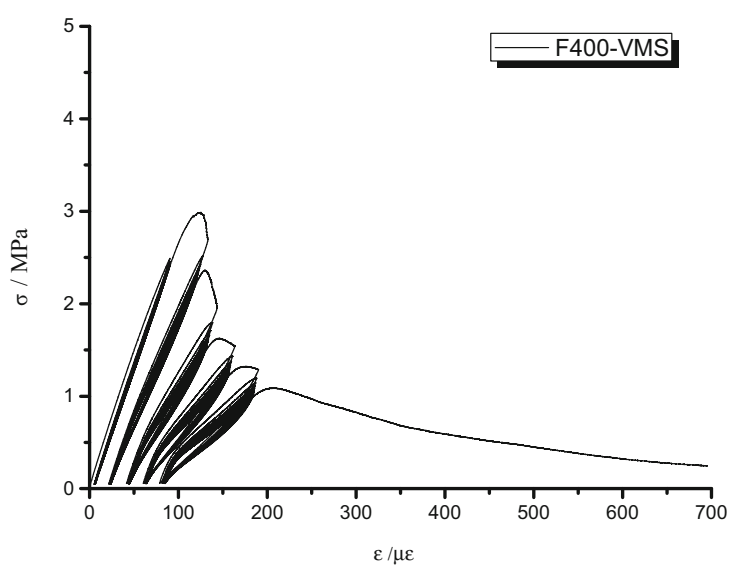

(d) F400

Figure 15. The stress-strain curves in the VMS test.

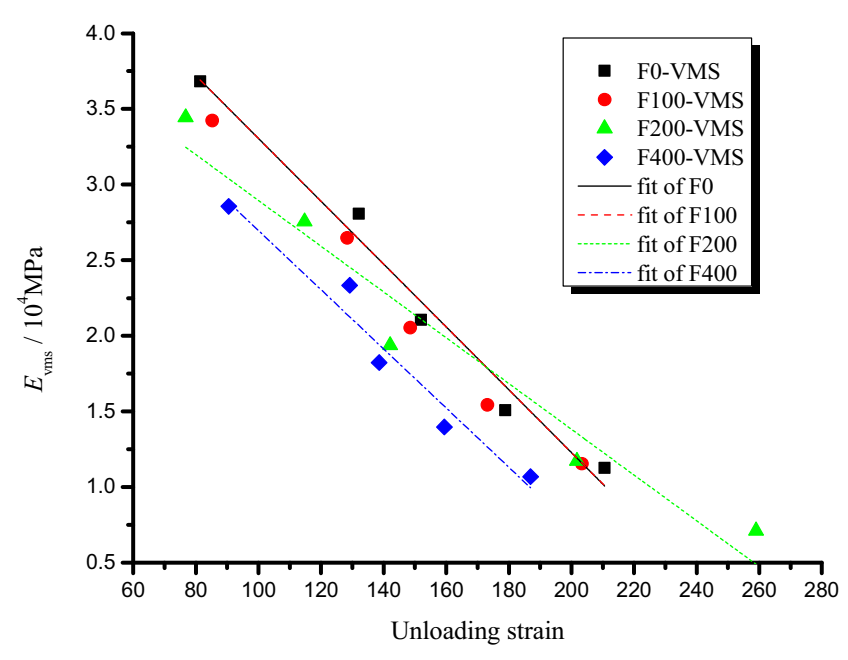

Figure 16. The curve of the first ring elastic modulus vs the unloading strain in the VMS test. modulus decreases gradually due to the frost damage, and the elastic modulus of specimens after 400 FTCs decreases significantly.

In the VMS test, the variations of the first ring elastic modulus in group F0, group F100 and group F200 are mainly similar. In group F400, the initial value of the first ring elastic modulus is much lower than in other groups. In this paper, there are two factors affecting the first ring elastic modulus: the freeze-thaw cycling and the cyclic loading. Under the limited FTCs, the microcracks increased with the limited growth and resulted in the initial $E_{v m s f}$ decrease. As the cyclic loading continued, the internal microcracks developed. The effect of the freeze-thaw cycling became less but the cyclic loading effect increased. It can be confirmed by the value change of the first ring elastic modulus in group F0, F100 and F200. The elastic moduli of these groups tended to be a certain value. Under the limited FTCs, the frost damage had great influence on 


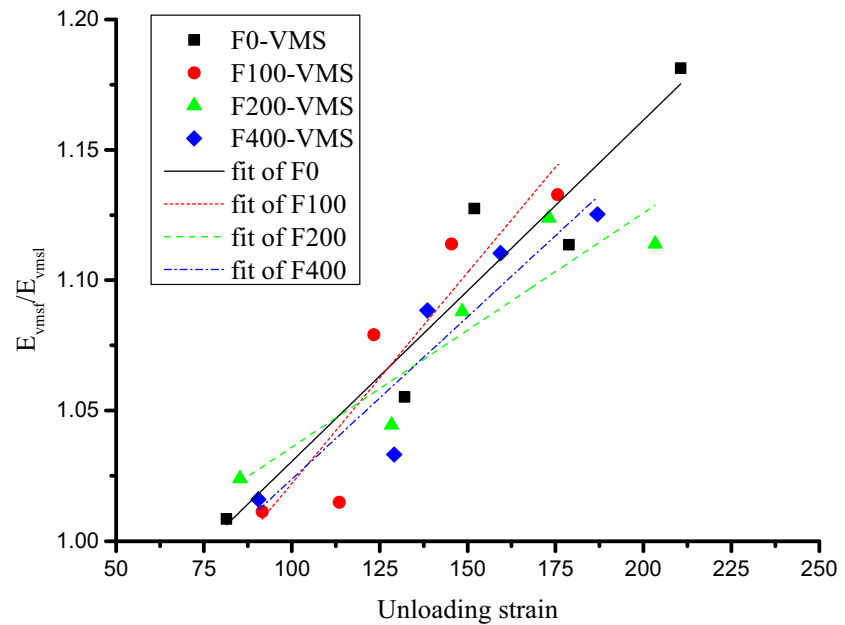

Figure 17. The curve of the elastic modulus ratio vs the unloading strain in the VMS test.

the initial value of the first ring elastic modulus but the final value mainly depended on the cyclic loading. Meanwhile, both the initial values of the first ring elastic modulus and the final values decreased in group F400. Later, the frost damage became the main factor of the elastic modulus variation in group F400. After the peak stress, a large number of microcracks rapidly developed and resulted in the rapid decrease of the first ring elastic modulus. The freeze-thaw cycling aggravated the material deterioration. Therefore, the mechanical properties of the material declined.

Figure 17 shows the relations between elastic modulus ratio $\left(E_{v m s f} / E_{v m s l}\right)$ and unloading strain. $E_{v m s f}$ is the elastic modulus of the first ring and $E_{v m s l}$ represents the elastic modulus of the last ring. Both of them were obtained from one strain level. All the values of elastic modulus ratio in this paper are over 1 . As figure 17 shows, while the unloading strain increases, the ratio increases. The elastic modulus ratio is related to the unloading strain. It is basically consistent for the elastic modulus ratio changing in the group F0, F100, F200 and F400. There is no apparent evidence to show the relation between the FTC and the elastic modulus ratio.

\subsection{Plastic deformation}

Plastic deformation is defined as the residual strain when the stress unloads to 0 . With the cyclic loading impacting, the plastic deformation gradually accumulated. As figures 13 and 15 present, the residual strain increased with the unloading strain getting large in the CSI test and the VMS test. Based on the curve of residual strain vs unloading strain after the peak stress, with increasing FTCs, the rising rate of the residual strain became large. Considering the microstructure variations, the reason can be
Table 2. The fitted value of parameters $a$ and $b$.

\begin{tabular}{lcl}
\hline Group number & $a$ & \multicolumn{1}{c}{$b$} \\
\hline F0-CSI & 0.699 & 42.87 \\
F100-CSI & 0.785 & 55.4 \\
F200-CSI & 0.794 & 61.052 \\
F400-CSI & 0.869 & 62.06 \\
F0-VMS & 0.649 & 46.76 \\
F100-VMS & 0.729 & 49.85 \\
F200-VMS & 0.820 & 71.86 \\
F400-VMS & 0.936 & 93.05 \\
\hline
\end{tabular}

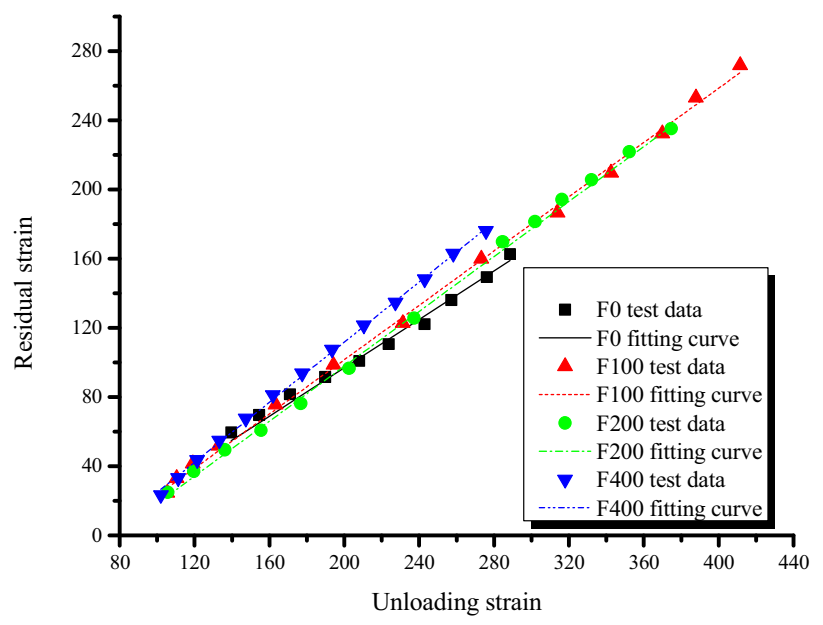

Figure 18. The curve of residual strain vs the unloading strain in the CSI test.

explained. Unfrozen concrete had a few cracks before loading. With loading, stress concentration caused rapid growth of individual cracks; however, the deformation of the individual cracks was limited. On the contrary, the frozen concrete had more internal cracks. After loading, all the cracks were fully stretched.

This paper raises a linear model to describe the relations between the residual strain and unloading strain, as follows:

$$
\varepsilon_{r}=a \varepsilon_{e u}-b
$$

where $\varepsilon_{r}$ is the residual strain and $\varepsilon_{e u}$ is the unloading strain; $a$ and $b$ are the coefficients related to FTC. Table 2 presents the fitted value of ' $a$ ' and ' $b$ '.

As figures 18 and 19 show, the linear model presents great fit for the post-peak test data. The unloading strain is composed of elastic deformation and irreversible plastic deformation. Under the same loading path, the coefficient ' $a$ ' represents the velocity of plastic deformation accumulation. Affected by frost damage, the value of $a$ gradually increases and tends to 1 . If the FTCs are more than the critical number, the value of $a$ reaches 1 , complete plastic deformation will happen and the capacity of concrete is completely lost. 


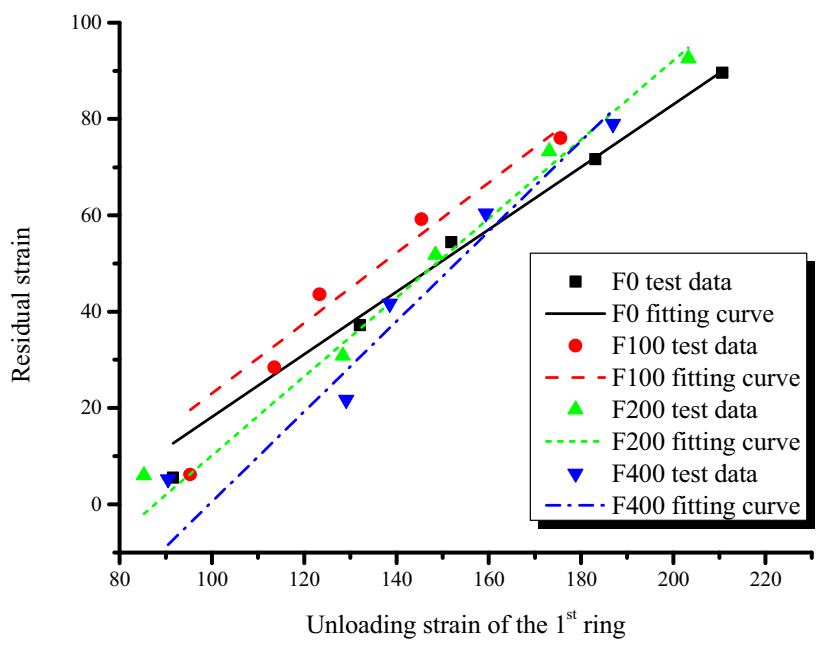

Figure 19. The curve of the residual strain vs the unloading strain in the VMS test.

\subsection{Dissipated energy per unit volume}

Figure 20 is the stress-strain curve of concrete in the CMS' test. As the figure shows, with the impact of the cyclic load,

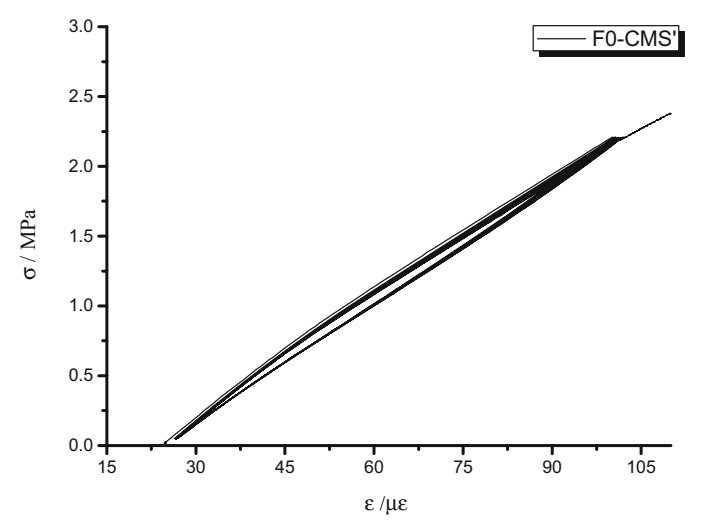

(a) F0

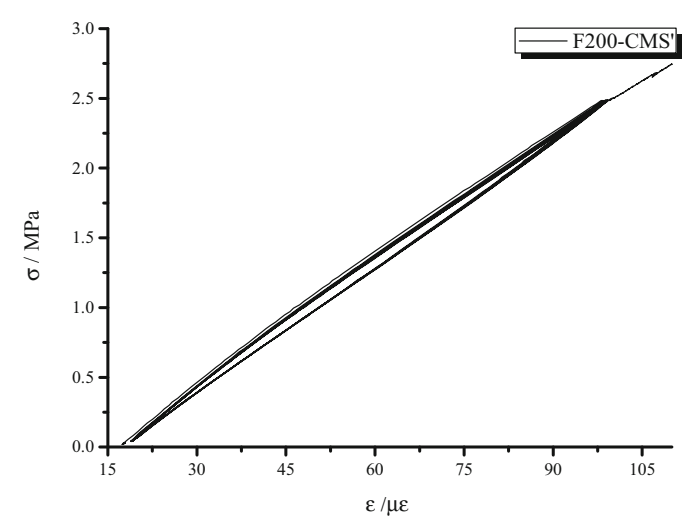

(c) F200 the hysteresis loops moved towards the direction of the strain increase. It means that the plastic deformation got large with continuing cyclic loading.

In the stress-strain curve of concrete, the unloading curve did not return along the path of the loading curve, the axial deformation could not be recovered and the unloading curve was always lower than the loading curve. Based on the previous study [27], the area under the loading curve in one cycle represents the unit volume energy produced by loading and corresponds to the area $S_{\text {load }}$ The area under the unloading curve in one cycle represents the unit volume energy released by unloading and corresponds to the area $S_{\text {unload. }}$. The difference between $S_{\text {load }}$ and $S_{\text {unload }}$ is equal to the area of hysteresis loop $S_{h}$, which is defined as the dissipated energy per unit volume. The area of $S_{\text {load }}$ and $S_{\text {unload }}$ can be divided into a number of small trapezoidal areas, and the area of hysteresis loop $S_{h}$ can be obtained by the integral method:

$$
S_{h}=S_{\text {load }}-S_{\text {unload }}=\sum_{\text {load }} \Delta S_{i}-\sum_{\text {unload }} \Delta S_{i}
$$

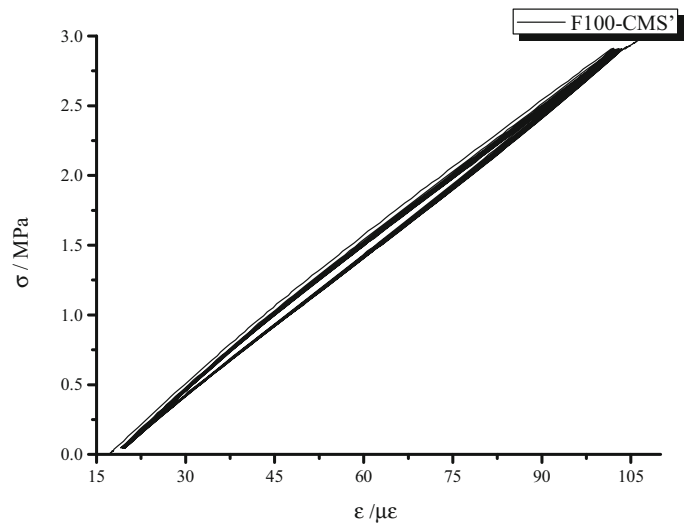

(b) F100

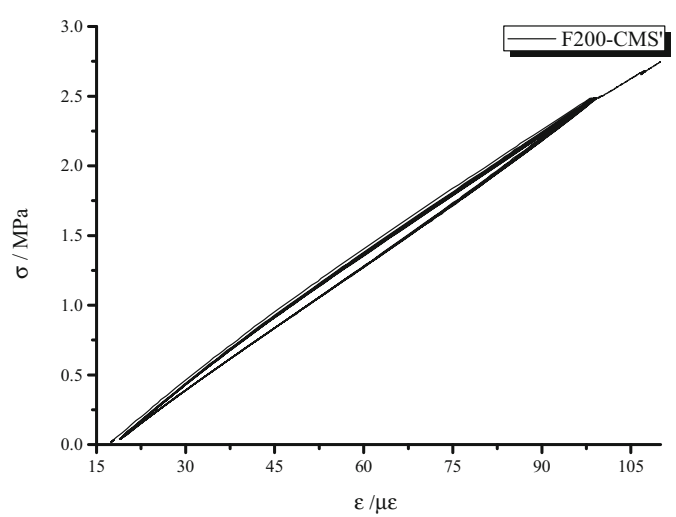

(d) F400

Figure 20. The stress-strain curves of concrete in the CMS' test. 


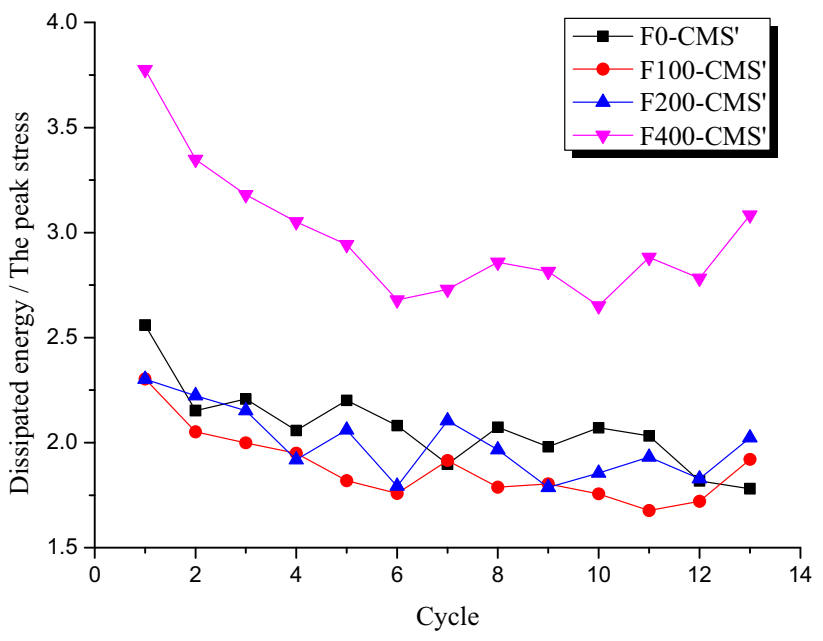

Figure 21. The relations between the dissipated energy per unit volume and the cycle after stress normalization.

As shown in figure 21, the dissipated energy per unit volume after stress normalization has a large decrease after the first cyclic loading. With increasing cycles, the unit volume dissipated energy tended to be stable. When stress rose over a certain value, the plastic deformation soared, and the internal cracks of the concrete grew rapidly, which led to the sharp decline of the energy released in the unloading process. After this, the energy produced in the loading process also declined. Eventually, the output and input energy achieved a relative balance.

In contrast to the results of specimens after different FTCs, the curves of the dissipated energy per unit volume in group F0, F100 and F200 are basically similar, but the curve of group F400 is much higher. After 400 FTCs, the material deterioration phenomenon appeared to be the increase of the plastic deformation and the change of released energy during the unloading process decreased. If the frost damage is over the critical limits, the concrete will get soft; the energy will rapidly accumulate inside the material and destroy the internal microstructure.

\section{Conclusions}

Physical and chemical damages will occur when concrete is subjected to the freeze-thaw cycling. The first criterion for physical damage is the mass loss rate. The air-entrained concrete used in this paper has experienced 400 FTCs and the mass loss rate is 0.5 , which shows that the mass loss is small and the result is satisfying. However, it was found by the SEM that with the micro-cracks in the concrete generated after freezing, the chemical properties of concrete began to change after 200 FTCs, and the microstructure of the material was destroyed after 400 FTCs. The compressive force promotes the closure of the micro-crack, and the pre-peak elastic modulus will be strengthened for a short time. This phenomenon does not exist under the tensile loading. Therefore, it is more dangerous for concrete subjected to the tensile force after frost. Compared with the compressive strength loss of ordinary concrete, the tensile strength of air-entrained concrete is still within a certain range after frost damage. Concrete is an elastic-plastic material. The elastic modulus variation shows that the initial elastic modulus of the material is reduced by frost damage, and, within 200 FTCs, the elastic modulus of specimens decreases a little. However, the elastic modulus of F400 decreased obviously. Meanwhile, the residual strain of concrete under cyclic loading was analysed, and the proportion of plastic deformation increased in the total deformation. The plastic deformation is sensitive to the frost damage. Based on the research about the energy dissipation of concrete after freeze-thaw cycling, it was shown that, within 200 FTCs, the energy dissipation of concrete fluctuated a little, and the dissipation energy increased obviously after 400 FTCs. Considering the result of the SEM test, the initial physical damage of the material was mainly manifested as the strength drop, the decrease of elastic modulus and the plastic enhancement. The chemical damage occurred in the later period, and the mechanical properties deteriorated rapidly. Moreover, the elastic modulus and the dissipation energy also changed greatly. In general, the frost damage will reduce the elastic deformation capacity of concrete, increase the proportion of plastic deformation in the total deformation, make the material crisp and then the concrete will lose the capacity.

\section{Acknowledgements}

The authors are grateful to the National Natural Science Foundation of China (Grant No. 51779085) and the Young Elite Scientists Sponsorship Program by CAST (Grant No. 2017QNRC001) for the financial support.

\section{References}

[1] Sun W, Zhang Y, Yan H and Mu R 1999 Damage and damage resistance of high strength concrete under the action of load and freeze-thaw cycles. Cement Concr. Res. 29: 1519-1523

[2] Kessler S, Thiel C, Grosse C and Gehlen C 2017 Effect of freeze-thaw damage on chloride ingress into concrete. Mater. Struct. 50: 121

[3] Jin M, Jiang L, Lu M, Xu N and Zhu Q 2017 Characterization of internal damage of concrete subjected to freezethaw cycles by electrochemical impedance spectroscopy. Constr. Build. Mater. 152: 702-707

[4] Hasan M, Ueda T and Sato Y 2008 Stress-strain relationship of frost-damaged concrete subjected to fatigue loading. ASCE J. Mater. Civ. Eng. 20: 37-45

[5] Penttala V 2006 Surface and internal deterioration of concrete due to saline and non-saline freeze-thaw loads. Cement Concr. Res. 36: 921-928 
[6] Xu S, Wang Y, Li A and Li Y 2015 Dynamic constitutive relation of concrete with freeze-thaw damage under repeated loading. J. Harbin Inst. Technol. (in Chinese) 47: 104-110

[7] $\mathrm{Yu} \mathrm{H}, \mathrm{Ma} \mathrm{H}$ and Yan K 2017 An equation for determining freeze-thaw fatigue damage in concrete and a model for predicting the service life. Constr. Build. Mater. 137: 104-116

[8] Duan A, Tian Y, Dai J and Jin W 2014 A stochastic damage model for evaluating the internal deterioration of concrete due to freeze-thaw action. Mater. Struct. 47: 1025-1039

[9] Hasan M, Okuyama H, Sato Y and Ueda T 2004 Stress-strain model of concrete damaged by freezing and thawing cycles. J. Adv. Concr. Technol. 2: 89-99

[10] Ray S and Kishen J 2012 Fatigue crack growth due to overloads in plain concrete using scaling laws. Sadhana 37: $107-124$

[11] Lund M, Hansen K, Brincker R, Jensen A and Amador S 2018 Evaluation of freeze-thaw durability of pervious concrete by use of operational modal analysis. Cement Concr. Res. 106: 57-64

[12] Al Rikabi F, Sargand S, Khoury I and Hussein H 2018 Material properties of synthetic fiber-reinforced concrete under freeze-thaw conditions. ASCE J. Mater. Civ. Eng. 30: 04018090

[13] Wu Y, Zhou X, Gao Y, Zhang L and Yang J 2019 Effect of soil variability on bearing capacity accounting for non-stationary characteristics of undrained shear strength. Comput. Geotech. 110: 199-210

[14] Du L and Folliard K 2005 Mechanisms of air entrainment in concrete. Cement Concr. Res. 35: 1463-1471

[15] Mayercsik N, Vandamme M and Kurtis K 2016 Assessing the efficiency of entrained air voids for freeze-thaw durability through modeling. Cement Concr. Res. 88: 43-59

[16] Sinha B, Gerstle K and Tulin L 1964 Stress-strain relations for concrete under cyclic loading. ACI J. Proc. 61: 195-212
[17] Maekawa K, Toongoenthong K, Gebreyouhannes E and Kishi T 2006 Direct path-integral scheme for fatigue simulation of reinforced concrete in shear. J. Adv. Concr. Technol. 4: 159-177

[18] El-Kashif K and Maekawa K 2004 Time-dependent nonlinearity of compression softening in concrete. J. Adv. Concr. Technol. 2: 233-247

[19] Tang X, An X and Maekawa K 2014 Behavior simulation model for SFRC and application to flexural fatigue in tension. J. Adv. Concr. Technol. 12: 352-362

[20] Chen X and Bu J 2016 Experimental study and modeling on direct tensile behavior of concrete under various loading regimes. ACI Mater. J. 113: 513-522

[21] Chen X, Bu J and Xu L 2016 Effect of strain rate on postpeak cyclic behavior of concrete in direct tension. Constr. Build. Mater. 124: 746-754

[22] Dyduch K, Szerszeń M and Destrebecq J 1994 Experimental investigation of the fatigue strength of plain concrete under high compressive loading. Mater. Struct. 27: 505-509

[23] Phillips D, Wu K and Zhang B 1996 Effects of loading frequency and stress reversal on fatigue life of plain concrete. Mag. Concr. Res. 48: 361-375

[24] Powers T C 1965 The mechanism of frost action in concrete. Stanton Walker Lecture Series on the Materials Science, Lecture No. 3, University of Maryland, p. 35

[25] Jian Y and Xing T 2006 Determination of dynamic elastic modulus of concrete using ultrasonic p-wave transducer. $J$. Build. Mater. (in Chinese) 9: 404-407

[26] Chen X, Xu L and Wu S 2015 Influence of pore structure on mechanical behavior of concrete under high strain rates. ASCE J. Mater. Civ. Eng. 28: 04015110

[27] Chen X, Huang Y, Chen C, Lu J and Fan X 2016 Experimental study and analytical modeling on hysteresis behavior of plain concrete in uniaxial cyclic tension. Int. J. Fatigue 96: $261-269$ 